\title{
Development of a Phenomenological Model On Surface Stabilized Ferroelectric Liquid Crystal Nanocomposite
}

\author{
Suman Kalyan Manna ${ }^{1}$, A.Sinha ${ }^{2}$ \\ ${ }^{1,2}$ (Department of Physics, Indian Institute of Technology Delhi, India)
}

\begin{abstract}
A liquid crystal nanocomposite, comprised of $5 w t \%$ ferroelectric type $\mathrm{BaTiO}_{3}$ nanoparticles in antiferroelectric phase liquid crystal host $M H P O B C$, has been prepared to study the effect of nanodope. Experimentally, it has been observed that the spontaneous polarization $\left(P_{s}\right)$ has been reduced and $\operatorname{SmC}{ }^{*}-S m A *$ transition temperature $\left(T^{*}\right)$ has been shifted towards the lower temperature side in case of $\mathrm{BaTiO}_{3}$ doped $M H P O B C$. In order to explain the above facts a new phenomenological model has been developed and formulated. Further studies on dielectric measurement and effect of higher doping concentration have satisfied the outcome of the model.
\end{abstract}

Keywords-barium titanate nanoparticles; nanowall; domain formation; Goldstone mode; system entropy

\section{Introduction}

Abrupt change in a characteristic property of any system is basically performed by change in constitute of the system. Such an abrupt change in response time from millisecond to microsecond was accomplished by replacing nematic phase liquid crystal with ferroelectric phase liquid crystal (FLC) [1]. Once such a selective constitute (FLC) mixed with appropriate nanoparticles, finer tuning of the characteristic property is possible through a certain way of interaction. As we are talking about liquid crystal display device, one of our prime concerns is response time. In surface stabilized ferroelectric liquid crystal (SSFLC) display device the response time is inversely proportional to the spontaneous polarization $\left(\mathrm{P}_{\mathrm{S}}\right)$ [2]. Doping nanoparticles in FLC can tune smoothly the value of $\mathrm{P}_{\mathrm{S}}$. Hence the change in $\mathrm{P}_{\mathrm{S}}$ by nano doping has been a subject of interest. Recent study has shown that the value of spontaneous polarization $\left(\mathrm{P}_{\mathrm{S}}\right)$ has been lowered for $\mathrm{BaTiO}_{3}$ doped FLC in compare to pure FLC. The possible reason mentioned [3] is due to antiparallel dipole-dipole correlation between molecules of the FLC mixture and barium titanate nanoparticles. In this paper our prime concern is to explain the reason of getting lowered the value of $\mathrm{P}_{\mathrm{S}}$ for doped FLC, for that we have made a nanocomposite of same dopant (i.e; ferroelectric $\mathrm{BaTiO}_{3}$ nps) doped in antiferroelectric liquid crystal host MHPOBC and studied on $\mathrm{P}_{\mathrm{S}}$ with varying temperature. Our experimental result also agrees the previous work that is the value of $P_{S}$ is decreased. Another remarkable outcome of the same experiment is that the transition temperature has been shifted to the lower side for doped sample in compare to pure sample. In order to understand physically the reason of decreasing the value of $\mathrm{P}_{S}$ and shifting the transition temperature towards lower side a new phenomenological model has been built up on the nanoparticles doped SSFLC device. Two experiments have been performed namely dielectric measurement and doping concentration variation agree the outcome of the formula, based on certain interaction energy.

\section{Materials}

Commercially available (99+\%, from Aldrich) $\mathrm{BaTiO}_{3}$ nanoparticles of size $<25 \mathrm{~nm}$ have been used. The phase transition temperature of $\mathrm{BaTiO}_{3}$ nanoparticls is particle size dependent. Experimentally it has been [4] shown that particle size $>100 \mathrm{~nm}$ has para-ferroelectric phase transition temperature is above $130^{\circ} \mathrm{C}$ but it becomes $150^{\circ} \mathrm{C}$ when the particle size is $72 \mathrm{~nm}$. Lesser the size of the particles transition temperature becomes higher and higher. The phase transition temperature of MHPOBC done by DSC is as below: $\mathrm{Cr}--84^{0} \mathrm{C}-\mathrm{SmC}_{\mathrm{A}}{ }^{*}-118.4^{0} \mathrm{C}-\mathrm{SmC}{ }_{\Upsilon}{ }^{*}-119.2^{0} \mathrm{C}-\mathrm{SmC}_{\beta}{ }^{*}-120.9^{0} \mathrm{C}--\mathrm{SmC}_{\alpha}{ }^{*}-122^{0} \mathrm{C}-\mathrm{SmA}-148^{0} \mathrm{C}$--Iso.

\section{Cell Filling}

To prepare doped MHPOBC, commercially available ferroelectric $\mathrm{BaTiO}_{3}$ nanoparticles were added $5 \mathrm{wt} \%$ in MHPOBC and then homogenized with ultrasonicator for 1hour. Two homogeneous cells , from INSTEC having thickness $4 \mu \mathrm{m}$ with effective area $1 \mathrm{~cm}^{2}$, were filled one with pure MHPOBC, and another with doped MHPOBC by capillary action at well above the clearing temperature $\left(>150^{\circ} \mathrm{C}\right)$ to fill homogeneously. 


\section{Instruments}

The dispersion was implemented by an ultrasonicator its operating frequency is $37 \mathrm{kHz}$. We used the heating stage (NISCO) rated as low, middle and high to provide the temperature well above the clearing temperature of liquid crystal at the time of cell filling. The phase sequence of MHPOBC was done by DSC. Polarizing microscope (OLYMPUS BX51) was used to make sure whether the sample was homogeneously filled or not. To determine the value of the spontaneous polarization, function generator (Tektronix AFG 3021) with varying voltage maximum of 10 volt $\mathrm{p}-\mathrm{p}$ and frequency range up to $25 \mathrm{MHz}$ was used. A programmable temperature controller (Instec STC200) was used to supply the scanning temperature ( T ) to liquid crystal cell attached to Instec LCH-S2 cell holder, was used to connect LC cell electrically instead of connecting wires directly. The cell holder was put in a Instec $\mathrm{HCP} 302$ hot and cold plate controlled by a programmable precision temperature controller graduated by $0.1^{\circ} \mathrm{C}$ can provide temperature ranging from $-190^{\circ} \mathrm{C}$ to $310^{\circ} \mathrm{C}$. The electric signal from the output of cell holder was fed to an oscilloscope (Tektronix TPS 2024). To measure the frequency dependent dielectric constant of the pure as well as doped sample Agilent LCR meter (Agilent E4980A), with frequency ranging from $20 \mathrm{~Hz}$ to $2 \mathrm{Mhz}$ was used.

\section{Experiments}

\section{a) Study of Micrographs}

Micrograph on dispersed sample after ultrasonication has been shown below. It is very clear from fig. 1 that there is no agglomeration. The long rod shape liquid crystal molecule and nanoparticles clusters are well separated. Two more micrographs in fig. 2 at room temperature (crystallography phase of LC), show that the cell fillings with pure sample and doped sample were done homogeneously.

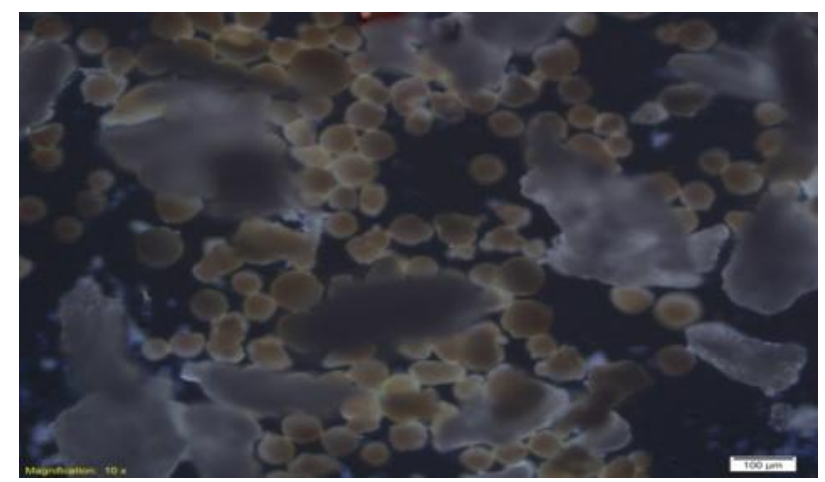

Fig. 1: Micrograph of $\mathrm{BaTiO}_{3}$ dispersed MHPOBC sample. Large rod shape geometry and small spherical shape geometry correspond to MHPOBC and clustered $\mathrm{BaTiO}_{3}$ respectively.

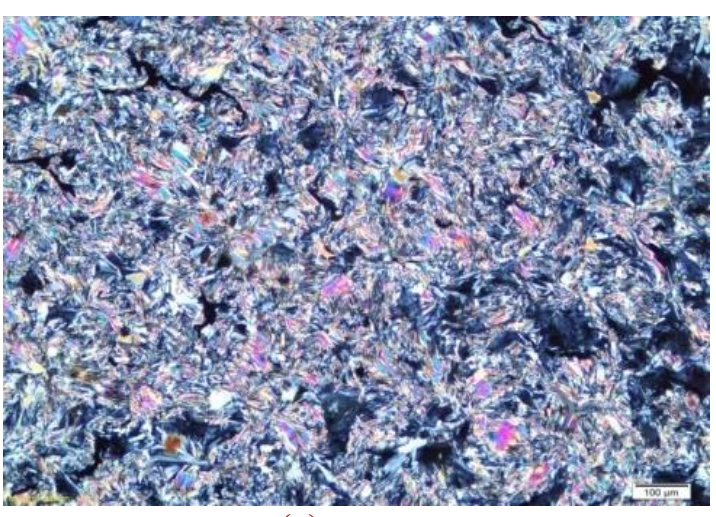

(a)

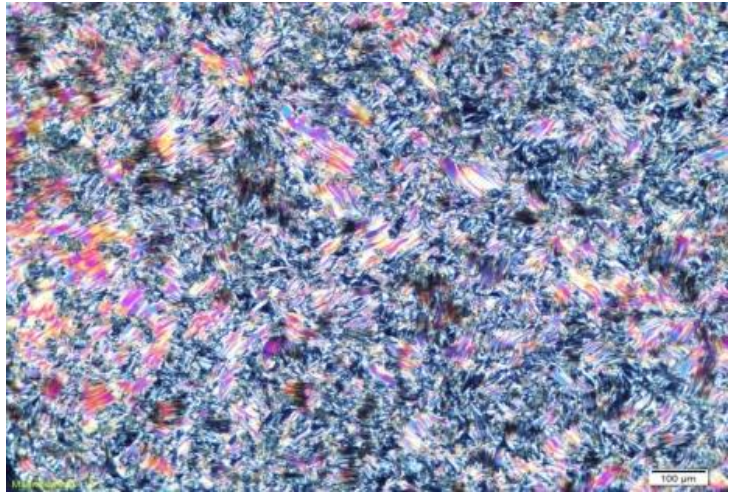

(b)

Fig.2: Micrographs of (a) pure (b) doped sample

b) Study of $\mathbf{P}_{S}$

Graphically $\mathrm{P}_{\mathrm{S}}$ is plotted over the entire temperature range for pure and doped sample. We can see in fig. 3 that the value of $P_{S}$ for pure sample is $49.1 \mathrm{nc} / \mathrm{cm}^{2}$ whereas for doped sample it is $20.8 \mathrm{nc} / \mathrm{cm}^{2}$. Fig.3 [a, b] further shows that $\mathrm{SmC}^{*}$ - SmA* transition temperature $\left(\mathrm{T}^{*}\right)$ has been shifted towards the lower side for doped sample. 


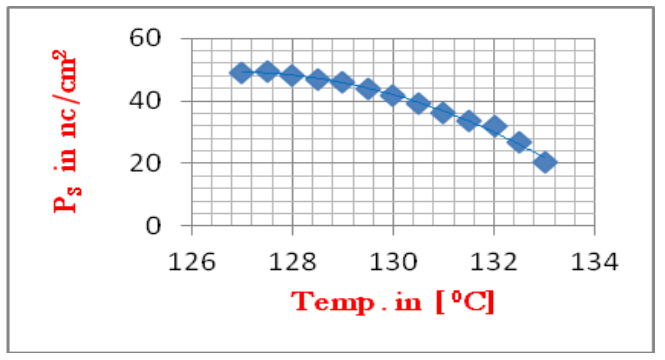

(a)

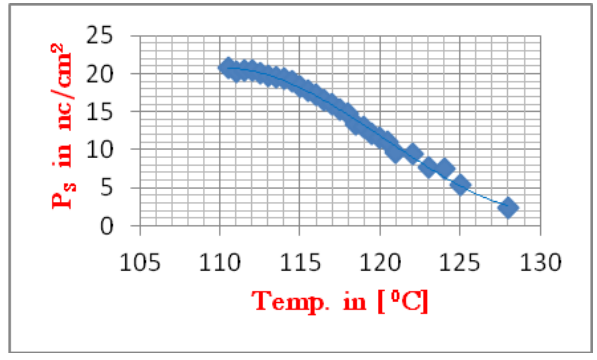

(b)

Fig.3: Shows spontaneous polarization vs. temperature obtained (a) for pure $\mathrm{MHPOBC}$ and (b) for $\mathrm{BaTiO}_{3}$ doped $\mathrm{MHPOBC}$.

For pure sample the transition temperature is projected on $134^{\circ} \mathrm{C}\left(=\mathrm{T}^{*}\right)$ whereas, for Doped Sample this transition temperature is $\left(\mathrm{T}^{*}=\right) 128^{\circ} \mathrm{C}$. In this occasion, a phenomenological model has been built up and further formulated on the light of Landau free energy expansion for better understanding and to explain the reason of shifting the transition temperature and lowering the value of spontaneous polarization.

\section{Phenomenological Model}

\section{a) Assumptions:}

i) The bookshelf structure is the building block of the SSFLC cell, where in each shelf (or Smectic layer) the LC molecules switches its polarization states under an external ac field. In between two such Smectic layers there is a spacing where there is no agitation. In such a system when we dope some nanoparticles the possibility of staying the nanoparticles can be assumed high in between the two Smectic layers.

ii) In the spacing, each nanoparticle will experience short range forces from the surrounding nanoparticles followed by the constraint of high dynamics (governed by continuous director fluctuation from $+\theta$ to $-\theta$ under an ac field) from both sides of spacing help to build a nano-wall, which can be treated as a thin ferroelectric layer.

iii) The nanoparticles remain in ferroelectric phase within the scanning temperature range of liquid crystal. So, we can write landau free energy expansion for thin ferroelectric type nano-wall by assuming $\mathbf{P}_{\mathbf{n}}$ as order parameter, which becomes zero when there is no external field and gets saturated to one when there is high electric field $\left(\mathbf{E}_{\mathbf{S}}\right)$.

\section{b) Model description}

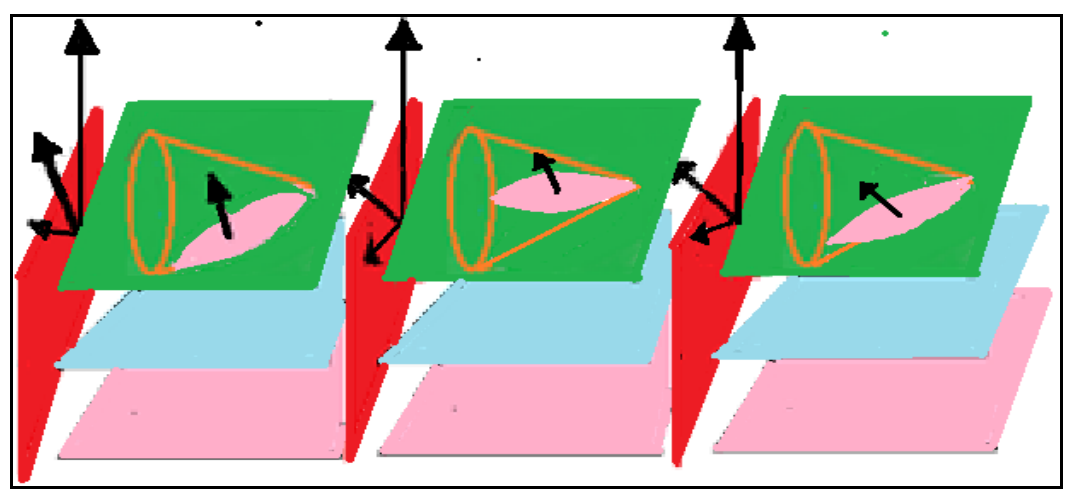

Fig.4: Model diagram, shows the azimuth angle $\varphi$ over a cone becomes random in direction

The above fig. 4 shows a schematic diagram of the phenomenological model. The red vertical plane in between any two smectic layers is the nano wall. Long arrow showing the instantaneous state of external electric field where small narrow one in the red plane shows the local field due to domain formation in ferroelectric nanowall. Thick arrow (in red plane) is the direction of the resultant electric field along which the dipole of LC

molecule will try to align which leads LC molecule to have some other azimuthal angle $\varphi$ than $\varphi_{0}$ (corresponds to undoped ideal system). The randomization in $\varphi$ basically reduce the spontaneous polarization of FLC. Unlike some other nematic phase liquid crystal nano-composite system, where insertion of nanoparticles helps LC matrix to be perfectly aligned due to dipole-dipole parallel interaction (results system order parameter increased 
hence the system entropy reduces), this system gets higher entropy which is manifested by randomization of the direction of $\varphi$ i.e; the strength of Goldstone mode [5] is increased.

Another outcome of the same experiment shows that due to nanodoping there is a reduction in $\mathrm{SmC}^{*}-\mathrm{SmA}$ * transition temperature. Physically this is obvious, as the system entropy (S) is increased, system automatically elevates itself to higher temperature $(T)$ so it will take less temperature $\left(T^{*}\right)$ further to change its phase. Basically at phase transition point free energy ( $\mathrm{F}=\mathrm{U}-\mathrm{TS}$ ) becomes zero. For a particular system internal energy $\mathrm{U}$ is fixed so the product T.S should be equal to $\mathrm{U}$ at phase transition point. If for a particular system $\mathrm{S}$ arises, $\mathrm{T}$ should be less to make the product T.S equals to $\mathrm{U}$.

\section{Formulation}

The above model can be formulated using Landau free energy expansion [6]. For the pure system, order parameter can be taken as spontaneous polarization $\left(\mathrm{P}_{\mathrm{S}}\right)$ which changes from maximum value in ferroelectric phase to zero in $\mathrm{SmA}^{*}$ phase upon changing the temperature.

$$
\mathrm{F}_{\mathrm{P}}=\frac{1}{2}\left(\mathrm{~T}^{*}-\mathrm{T}\right) \mathrm{P}_{\mathrm{S}}^{2}+\frac{1}{3} \mathrm{BP}_{\mathrm{S}}^{3}-\mathrm{P}_{\mathrm{S}} \mathrm{E}
$$

Where, $F_{p}$ is the free energy available to the system as output. Third term is the interaction energy in presence of external electric field. We will be taking this interaction energy as negative as it is treated as input to the LC system. By nano doping we are basically giving some disturbance to the pure LC system. This disturbance should be taken also as negative quantity as we are giving it from externally as an input to the pure system. So in the presence of such a disturbance the total free energy of the composite system is:

$$
\mathrm{F}_{\mathrm{P}}=\frac{1}{2}\left(\mathrm{~T}^{*}-\mathrm{T}\right) \mathrm{P}_{\mathrm{S}}{ }^{2}+\frac{1}{3} \mathrm{BP}_{\mathrm{S}}{ }^{3}-\mathrm{P}_{\mathrm{S}} \mathrm{E}-\text { interaction energy }\left[\mathrm{F}_{\mathrm{D}}\right]
$$

Electric field dependence disturbance can be written as

$$
f_{D}=\frac{1}{2}\left(\mathrm{E}_{\mathrm{S}}-\mathrm{E}\right) \mathrm{P}_{\mathrm{N}}{ }^{2}+\frac{1}{3} \mathrm{~B}^{\prime} \mathrm{P}_{\mathrm{N}}{ }^{3}
$$

where $\mathrm{E}$ is the varying electric field and $\mathrm{E}_{\mathrm{S}}$, (turning point) is the saturated electric field where all ferroelectric domains become perfectly aligned to applied field [i.e; order parameter becomes 1]. $\mathbf{P}_{\mathbf{N}}$ is the spontaneous polarization and, can be taken as the order parameter of nano-wall. As the disturbance is mediated by $\varphi$ to the LC system, we can write the interaction energy due to NPs insertion as

$$
\mathrm{F}_{\mathrm{D}}=f_{D} \cdot \varphi_{\mathrm{a}}
$$

Where $\varphi_{\mathrm{a}}$ is the average of $\varphi$. The total energy can be written as:

Putting $\mathrm{P}_{\mathrm{S}}=\chi \mathrm{E}$ in the above equation

$$
\mathrm{F}=\frac{1}{2}\left(\mathrm{~T}^{*}-\mathrm{T}\right) \mathrm{P}_{\mathrm{S}}^{2}+\frac{1}{3} \mathrm{BP}_{\mathrm{S}}^{3}-\mathrm{P}_{\mathrm{S}} \mathrm{E}-\left[\frac{1}{2}\left(\mathrm{E}_{\mathrm{S}}-\mathrm{E}\right) \mathrm{P}_{\mathrm{N}}{ }^{2}+\frac{1}{3} \mathrm{~B}^{\prime} \mathrm{P}_{\mathrm{N}}^{3}\right] \cdot \varphi_{a}
$$

$$
\begin{aligned}
\mathrm{F} & =\frac{1}{2}\left(\mathrm{~T}^{*}-\mathrm{T}\right) \mathrm{P}_{\mathrm{S}}^{2}+\frac{1}{3} \mathrm{BP}_{\mathrm{S}}^{3}-\mathrm{P}_{\mathrm{S}} \mathrm{E}-\left[\frac{1}{2}\left(\mathrm{E}_{\mathrm{S}}-\frac{\mathrm{P}_{\mathrm{S}}}{\chi}\right) \mathrm{P}_{\mathrm{N}}{ }^{2}+\frac{1}{3} \mathrm{~B}^{\prime} \mathrm{P}_{\mathrm{N}}^{3}\right] \cdot \varphi_{a} \\
\mathrm{Or}, \quad \mathrm{F} & =\frac{1}{2}\left(\mathrm{~T}^{*}-\mathrm{T}\right) \mathrm{P}_{\mathrm{S}}^{2}+\frac{1}{3} \mathrm{BP}_{\mathrm{S}}^{3}-\mathrm{P}_{\mathrm{S}} \mathrm{E}-\frac{1}{2} \mathrm{E}_{\mathrm{S}} \mathrm{P}_{\mathrm{N}}^{2} \varphi_{a}+\frac{1}{2} \frac{\mathrm{P}_{\mathrm{S}}}{\chi} \mathrm{P}_{\mathrm{N}}^{2} \varphi_{a}-\frac{1}{3} \mathrm{~B}^{\prime} \mathrm{P}_{\mathrm{N}}^{3} \varphi_{a}
\end{aligned}
$$

In equilibrium, the function $\mathrm{F}\left(\mathrm{T}, \mathrm{P}_{\mathrm{S}}, \mathrm{P}_{\mathrm{N}}\right)$ must have a minimum value, therefore at any Temperature $\mathrm{dF} / \mathrm{dP}_{\mathrm{N}}=0$ and, $\mathrm{d}^{2} \mathrm{~F} / \mathrm{dP}_{\mathrm{N}}^{2}>0$

So, $d F / d P_{N}=-E_{S}+\frac{P_{S}}{\chi}-B^{\prime} P_{N}=0$

Or, $\mathbf{P}_{\mathrm{N}}=\frac{1}{\mathrm{~B}^{\prime}}\left(\frac{\mathbf{P}_{\mathrm{S}}}{\chi}-\mathbf{E}_{\mathrm{S}}\right)$

Putting the above value of $\mathrm{P}_{\mathrm{N}}$ in eqn.5 and arranging the co-efficients of $\mathrm{P}_{\mathrm{S}}, \mathrm{P}_{\mathrm{S}}{ }^{2}, \mathrm{P}_{\mathrm{S}}{ }^{3}$ :

$$
\mathrm{F}=-\frac{\mathrm{E}_{\mathrm{S}}^{3} \varphi_{a}}{6 \mathrm{~B}^{\prime 2}}+\left(\frac{\varphi_{a} \mathrm{E}_{\mathrm{S}}^{2}}{2 \chi \mathrm{B}^{\prime 2}}-\mathrm{E}\right) \mathrm{P}_{\mathrm{S}}+\frac{1}{2}\left(\mathrm{~T}^{*}-\frac{\mathrm{E}_{\mathrm{S}} \varphi_{a}}{\mathrm{~B}^{\prime 2} \chi^{2}}-\mathrm{T}\right) \mathrm{P}_{\mathrm{S}}^{2}+\frac{1}{3}\left(\mathrm{~B}+\frac{\varphi_{a}}{2 \chi^{3} \mathrm{~B}^{\prime 2}}\right) \mathrm{P}_{\mathrm{S}}^{3}
$$

Eqn.7, shows that the transition temperature $\mathrm{T}^{*}$ now reduced by the factor $\frac{\mathrm{E}_{\mathrm{s}} \varphi_{a}}{\mathrm{~B}^{2} \chi^{2}}$ where $\varphi_{\mathrm{a}}$ is only variable quantity which has further dependency on

1) applied electric field

2) doping concentration 
Development of a Phenomenological Model on Surface Stabilized Ferroelectric Liquid Crystal

It seems to be quite reasonable that by increasing the field strength the doped system moves toward the pure one [as the domains in ferroelectric nanowall will be trying to align more along the external field results distribution in $\varphi_{\mathrm{a}}$ becomes narrower i.e; entropy reduces] hence transition temperature gets higher value. Another control on transition temperature is doping concentration. Increasing the doping concentration basically allows the system to move away from the pure system, resulting lower transition temperature as system entropy increases. If we want to verify eqn.7 with respect to system stability, we take first derivative of eqn.7

$$
\begin{aligned}
& \frac{\mathrm{dF}}{\mathrm{dP}_{\mathrm{S}}}=\left(\frac{\varphi_{a} \mathrm{E}_{\mathrm{S}}^{2}}{2 \chi \mathrm{B}^{\prime 2}}-\mathrm{E}\right)+\left(\left(\mathrm{T}^{*}-\frac{\mathrm{E}_{\mathrm{S}} \varphi_{a}}{\mathrm{~B}^{\prime 2} \chi^{2}}\right)-\mathrm{T}\right) \mathrm{P}_{\mathrm{S}}+\left(\mathrm{B}+\frac{1}{2} \frac{\varphi_{a}}{\chi^{3} \mathrm{~B}^{\prime 2}}\right) \mathrm{P}_{\mathrm{S}}^{2}=0 \\
& \text { Or, }\left.\frac{\mathrm{dF}}{\mathrm{dP}_{\mathrm{S}}}\right|_{\mathrm{E}=0}=\frac{\varphi_{a} \mathrm{E}_{\mathrm{S}}^{2}}{2 \chi \mathrm{B}^{\prime 2}}=0
\end{aligned}
$$

When $\mathrm{E}=0$ it seems that system does not obeying stability condition as in left hand side only the remaining quantity $\frac{\varphi_{a} \mathrm{E}_{\mathrm{s}}{ }^{2}}{2 \chi \mathrm{B}^{2}}$ does not vanish (rests all are zero as $\mathrm{P}_{\mathrm{S}}=0$ when $\mathrm{E}=0$ ). But, if we argue the situation when there is no external electric field $(\mathrm{E}=0)$ the ferroelectric domains in the nanowall will become completely disordered, which prevails randomness to the $\varphi_{\mathrm{a}}$, upon average gives zero.

\section{Experimental Verification}

\section{1) Dielectric measurement}

Dielectric measurement was performed on both for pure sample as well as doped sample. The imaginary part of dielectric constant which represents the amount of energy absorbed by any process to be happened inside the system is shown on a fixed range of frequency scale with increasing DC bias voltage. Fig.5a shows that the Goldstone mode for pure sample diminished by 4 volts DC bias, whereas fig.5b shows that the Goldstone mode has been diminished at higher DC bias voltage ( 7 volts).

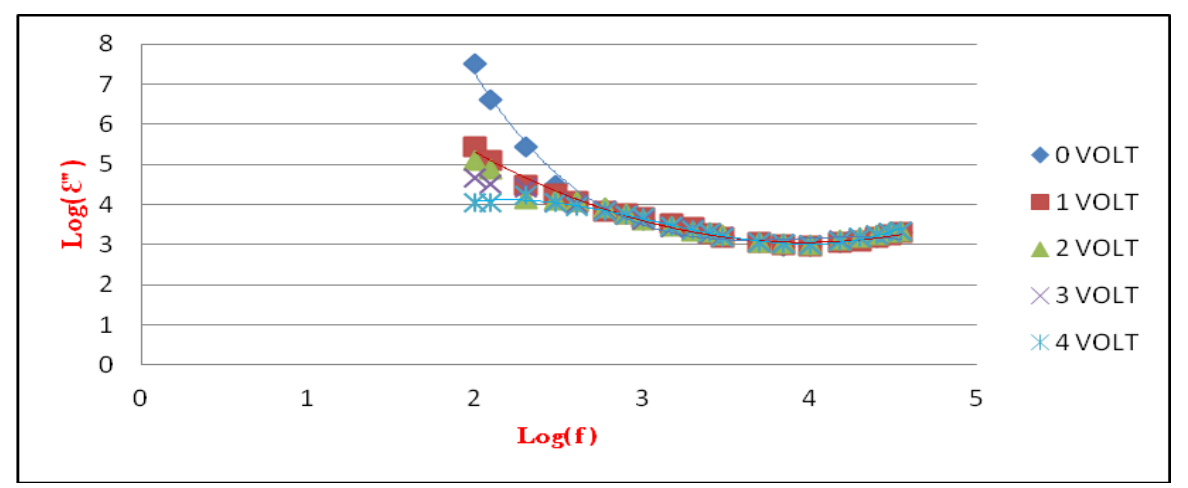

Fig.5a: Shows the change in relaxation peak of Goldstone mode over a fixed range of frequency with increasing DC bias for pure sample.

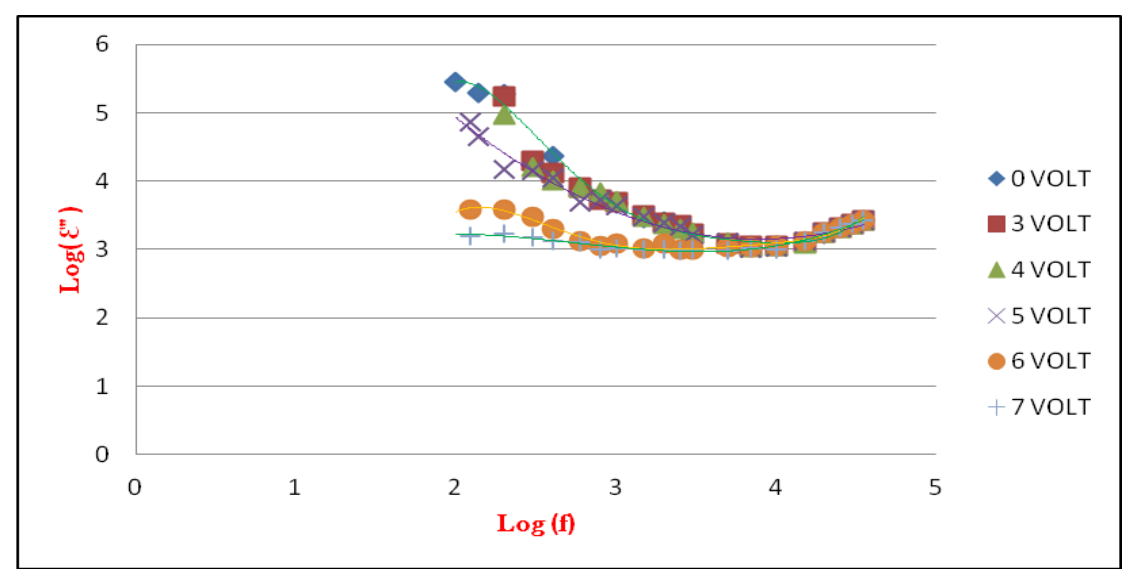


Fig.5b: Shows that the change in relaxation peak of Goldstone mode over a fixed frequency range with increasing DC bias for doped sample.

As Goldstone mode is due to fluctuation in $\varphi$, the higher voltage is needed to stop this mode for doped sample may be inferred that an extra amount of fluctuation in $\varphi$ has been produced due to nanodope.

\section{2) Effect of high doping concentration}

High doping ( $7 \mathrm{wt} \%$ ) reduced further (fig.6) the transition temperature ( $\left.\mathrm{T}^{*}=121\right)$. As more concentration of nanodope induces system entropy higher.

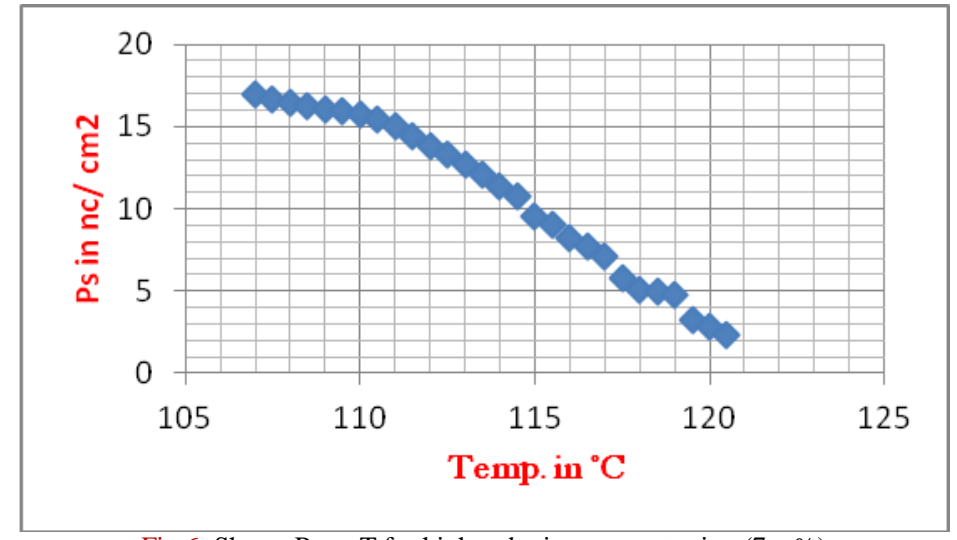

Fig.6: Shows Ps vs.T for higher doping concentration (7wt $\%)$

\section{Conclusion}

From dielectric measurement it can be inferred that the nanodope mainly affects the Gold-Stone mode. The randomness (or fluctuation) in $\varphi$ for pure system can be removed at the cost of relatively lower voltage but, for doped sample as the $\varphi$ randomness increases, system takes more voltage to decouple the $\varphi$ fluctuation.

The model has also been verified by increasing the doping concentration, leads entropy of the system to be raised hence the role of temperature, to make free energy $(\mathbf{F})$ of the composite system to be zero, becomes less i.e; transition temperature is reduced.

In Landau expansion for free energy there is $\mathrm{T}^{*}$ which basically serves as an indication that if we start scanning the temperature we push the system to some potential level where it becomes unable to give any energy out. Relating with the thermodynamic basic free energy equation $\mathrm{F}=\mathrm{U}-\mathrm{ST}$ it is quite obvious to treat $\mathrm{T}^{*}$ as the internal energy $(\mathrm{U})$ of the system. In this occasion, we have taken $\left(\mathrm{T}^{*} \mathrm{~T}\right)$ instead of $\left(\mathrm{T}-\mathrm{T}^{*}\right)$ in all the Landau expansions used (only for better intuition) in this paper but, it does not degrade the generality as we have taken this convention to write pure system energy $\left(\mathbf{F}_{\mathbf{P}}\right)$ as well as interaction energy $\left(f_{D}\right)$.

\section{References}

[1]. Hao-Hsun Liang, Ya-Zhi Xiao, Fu-Jhen Hsh, Che-Cheng Wu and Jiunm-Yih Lee, 'Enhancing the electro-optical properties of ferroelectric liquid crystals by doping ferroelectric nanoparticles', Taylor \& Francis, Liquid Crystals, Vol.37, No.3, 2010.

T.Joshi, A.Kumar, J.Prakash, and A.M.Biradar, ' Low power operation of ferroelectric liquid crystal system displayed with zinc oxide nanoparticles', Applied Physics Letters, 96, 2010.

[2]. A.Mikulko, P.Arora, A.Glushchenko, A.Lapanik and W.Haase, 'Complementary studies of $\mathrm{BaTiO}_{3}$ nanoparticles suspended in a ferroelectric liquid- crystalline mixture', IOP Science, EPL, 87, 2009.

[3]. Millicent B.Smith, Katharine Page, Theo Siegrist, Peter L.Redmond, Erich C.Walter, Ram Seshadri, Louis E.Brus, and Michael L.Steigerwald, 'Crystal Structure and the Paraelectric-to-Ferroelectric Phase Transition of Nanoscale BaTiO ${ }_{3}$ ', JACS Articles, 2008.

[4]. S.U.Vallerien, F.Kremer, H.Kapitza, R.Zentel, W.Frank, ' Field Dependent Soft and Goldstone Mode in a Ferroelectric Liquid Crystal as Studied by Dielectric Spectroscopy', Physics Letters A, Vol.138, No.4,5,1989.

[5]. Lev M.Blinov, 'Structure and Properties of Liquid Crystals', Springer New York, 2011. 